\title{
A GREEN FUNCTION ESTIMATION OF CORRECTION TO VEGARD'S LAW FOR ISOVALENT SUBSTITUTIONAL DEFECTS IN ALKALI HALIDE CRYSTALS
}

\author{
S. Datta Roy (PaUl) ${ }^{a}$ and S.K. Das ${ }^{b}$ \\ ${ }^{a}$ Department of Physics, Bethune College, Calcutta 700 006, India \\ ${ }^{b}$ Azad Physics Centre, Department of Physics \\ Maulana Azad College, Calcutta 700 013, India
}

(Received October 25, 1999; revised version January 25, 2000)

\begin{abstract}
A Green function method is developed for estimation of lattice parameter $r(\chi)$ of an $\mathrm{AX}-\mathrm{BX}$ solid solution over entire composition range $(\chi=0$ to 1 ). The $r(\chi)$ obtained in the present method is different from the value $r_{\mathrm{V}}(\chi)$ suggested by Vegard's law. We estimated the deviation from Vegard's law, $r(\chi)-r_{\mathrm{V}}(\chi)$, over entire composition range for isovalent substitutional defect in alkali halide crystals. When compared to X-ray diffraction measurements, we find that the deviation is in correct direction, variation of $r(\chi)$ with $\chi$ is consistent with experiment and the overall agreement is fairly good considering the accuracy of the experiment.
\end{abstract}

PACS numbers: $61.72 . \mathrm{Bb}, 61.82 . \mathrm{Bg}$

\section{Introduction}

From early works on equilibrium diagram it is clear that the first addition of one binary compound to another binary compound does not produce a new phase, but leads to a homogeneous alloy which is regarded as a solid solution of one binary compound in the other. X-ray diffraction photographs show that the general pattern of the lines or spots remain the same both for the solid solvent and for the solid solutions but the exact position of the lines or spots differ slightly for the two cases. These investigations indicate that the solid solution has the same structure as the parent crystal and formation of substitutional solid solution is accompanied with a uniform lattice relaxation, either contraction or expansion.

L. Vegard [1] was first to propose a law to fit the observed lattice parameter change. He originally deduced it for solid solution in ionic crystals and later 
extended that for metallic solid solutions also. If the lattice parameter for the observed AX-BX solid solution is denoted by $a(\chi)$ and that of the constituents by $a_{\mathrm{AX}}$ and $a_{\mathrm{BX}}$, then Vegard's law states that

$$
a(\chi)=(1-\chi) a_{\mathrm{AX}}+\chi a_{\mathrm{BX}},
$$

where $\chi$ is the molar fraction of $\mathrm{BX}$ in the $\mathrm{AX}-\mathrm{BX}$ solid solution.

Extended X-ray absorption fine structure (EXAFS) measurements [2], on the other hand, indicate a different picture. These measurements provide a wide range of information regarding nearest neighbour (nn) distances in binary solid solutions over entire composition range. They observed for an $\mathrm{AX}-\mathrm{BX}$ solid solution, at any defect concentration $\chi$, that the first neighbour separations between the ion pairs $\mathrm{A} \leftrightarrow \mathrm{X}$ and $\mathrm{B} \leftrightarrow \mathrm{X}$ differ substantially from each other and also from the value suggested by Vegard's law. Thus a striking difference is apparent between the experimental evidences available from the two types of experiments for relaxation.

Early theoretical estimates of the relaxation are mostly on dilute concentration of defects (i.e. $\chi \rightarrow 0$ or $\chi \rightarrow 1$ ) and thus the concerned authors could not trace the above problem in their investigations for obvious reason.

For high concentration of defects, different approaches, like Durham et al. [3] from atomistic calculation, Hardy et al. [4] from lattice static calculation, Fukai [5] from both semidiscrete and superlattice method, Fancher and Barsch [6] from semidiscrete method and present authors [7] from a self-consistent semidiscrete method, obtained a single value of relaxation for both types of nearest neighbour pairs indicating existence of a virtual crystal having lattice parameter $r(\chi)$, fairly close to the values suggested by Vegard's law.

In recent past we have developed a Green function technique [8] to evaluate the nearest neighbour separation of ion pairs $A \leftrightarrow X$ and $B \leftrightarrow X$ for an $A X-B X$ solid solution over entire composition range. Values obtained from this model, in case of alkali halide solid solutions, are found to have remarkably good agreement with EXAFS measurements [2].

In the present work, we have extended this Green function technique to find the virtual crystal nearest neighbour separation $r(\chi)$ in terms of the $\mathrm{nn}$ ion pair separation $r_{\mathrm{A} \rightarrow \mathrm{X}}$ and $r_{\mathrm{B} \leftrightarrow \mathrm{X}}$, corresponding to some defect concentration $\chi$. It is interesting to note that Vegard's law suggests a linear dependence of $\chi$ for virtual crystal lattice parameter $r(\chi)$, whereas the present model finds a quadratic dependence of $r(\chi)$ on $\chi$ along with a linear dependence, which seems to be quite significant from the non-linear nature of the experimental curves, for all available alkali halide solid solutions. We have plotted deviation from the Vegard law, $r(\chi)-r_{\mathrm{V}}$, from the present model and compared with experimental values for four types of alkali halide solid solutions. For each case the deviation is in the right direction and the agreement is also fairly encouraging considering the simplicity of the model. Easy handling opportunity makes the model suitable for solid solutions having complicated structures.

The model is already tested for dilute metallic solid solutions [9] for which we have got good agreement with the experimental results. 


\section{Theory}

In a solid solution $\mathrm{AX}-\mathrm{BX}$, at any stage of composition $\mathrm{A}_{1-\chi} \mathrm{B}_{\chi} \mathrm{X}$, if any experiment (e.g. X-ray diffraction experiments) fails to measure the individual relaxations at different sites but yields a relaxation picture on the average, then for this average lattice or virtual lattice, we propose a lattice parameter $r(\chi)$ (nn separation) given by

$$
r(\chi)=\chi r_{\mathrm{B} \leftrightarrow \mathrm{X}}(\chi)+(1-\chi) r_{\mathrm{A} \leftrightarrow \mathrm{X}}(1-\chi),
$$

where $r_{\mathrm{B} \leftrightarrow \mathrm{X}}(\chi)$ and $r_{\mathrm{A} \leftrightarrow \mathrm{X}}(1-\chi)$ are the nearest neighbour separations between the ion pairs $B \leftrightarrow X$ and $A \leftrightarrow X$, respectively, measured (EXAFS measurements) at the same composition stage.

For a finite concentration $\chi$, we consider the concentration to be achieved by successive addition of a single defect. As a result $r_{\mathrm{A} \leftrightarrow \mathrm{X}}$ and $r_{\mathrm{B} \leftrightarrow \mathrm{X}}$ will be changing continually, gradually reaching $r_{\mathrm{A} \leftrightarrow \mathrm{X}}(1-\chi)$ and $r_{\mathrm{B} \leftrightarrow \mathrm{X}}(\chi)$, respectively. If in an $\mathrm{AX}$ host crystal a guest ion $B$ replaces an isovalent ion $A$, then due this replacement the $\mathrm{X}$ ion, which is nearest neighbour to the defect ion $\mathrm{B}$, relaxes by $u_{1}$ (say). Following Huang's [10] idea of uniform dilation, we apply dilute concentration relaxation $u_{1}$ to find $r_{\mathrm{B} \leftrightarrow \mathrm{x}}(\chi)$, and obtain

$$
r_{\mathrm{B}-\mathrm{X}}(\chi)=r_{1}\left(1+\frac{4 \pi K_{1} r_{1} \chi}{3 v_{1}}\right)+u_{1}
$$

where $r_{1}, v_{1}$ are the $\mathrm{nn}$ separation and atomic volume, respectively, of the perfect AX crystal, $\chi$ - the mole fraction of defect ion $\mathrm{B}$ and $K_{1}$ - the defect strength constant. Similarly, starting from a perfect BX crystal and replacing $\chi^{\prime}[=(1-\chi)]$ mole fraction of $\mathrm{B}$-ion by isovalent defect ion $\mathrm{A}$, we obtain

$$
r_{\mathrm{A} \leftrightarrow \mathrm{X}}\left(\chi^{\prime}\right)=r_{2}\left(1+\frac{4 \pi K_{2} r_{2} \chi^{\prime}}{3 v_{2}}\right)+u_{2},
$$

where $u_{2}$ is the dilute concentration relaxation of $\mathrm{X}$ ion which is nearest neighbour to defect ion $\mathrm{A}, r_{2}$ and $v_{2}$ are the $\mathrm{nn}$ separation and atomic volume, respectively, of the perfect BX crystal and $K_{2}$ is the defect strength constant.

For estimation of defect strength constant we refer to our work [8], where a smooth merging of the microscopic relaxation, experienced by the near neighbourhood of the defect ion, to the macroscopic strain developed in the further region of the crystal, yields

$$
K_{1}=k_{1} u_{1} r_{1}^{2}
$$

and

$$
K_{2}=k_{2} u_{2} r_{2}^{2} \text {, }
$$

where $k_{1}$ and $k_{2}$ are constants to be determined from the boundary conditions. From Eqs. (5), (6) and (3), (4), we get

$$
r_{\mathrm{B} \leftrightarrow \mathrm{X}}(\chi)=r_{1}+\frac{4 \pi k_{1} r_{1}^{3} u_{1} \chi}{3 v_{1}}+u_{1}
$$

and

$$
r_{\mathrm{A} \rightarrow \mathrm{X}}\left(\chi^{\prime}\right)=r_{2}+\frac{4 \pi k_{2} r_{2}^{3} u_{2} \chi^{\prime}}{3 v_{2}}+u_{2}
$$


For NaCl-type structure, we get from (5) and (6)

$$
\begin{aligned}
& r_{\mathrm{B} \leftrightarrow \mathrm{X}}(\chi)=r_{1}+\frac{2 \pi k_{1} u_{1} \chi}{3}+u_{1}, \\
& r_{\mathrm{A} \leftrightarrow \mathrm{X}}\left(\chi^{\prime}\right)=r_{2}+\frac{2 \pi k_{2} u_{2} \chi^{\prime}}{3}+u_{2} .
\end{aligned}
$$

For a $\mathrm{A}_{1-\chi} \mathrm{B}_{\chi} \mathrm{X}$ solid solution $\chi^{\prime}=1-\chi$ and the boundary conditions are (I) at $\chi=1, r_{\mathrm{B} \leftrightarrow \mathrm{X}}(\chi)=r_{2}$ and we get

$$
k_{1}=\frac{1.5}{\pi}\left(-1+\frac{r_{2}-r_{1}}{u_{1}}\right) \text {, }
$$

and (II) at $\chi^{\prime}=1, r_{\mathrm{A} \leftrightarrow \mathrm{X}}\left(\chi^{\prime}\right)=r_{1}$ we get

$$
k_{2}=\frac{1.5}{\pi}\left(-1+\frac{r_{1}-r_{2}}{u_{2}}\right) \text {. }
$$

Substituting (11) and (12) in (9) and (10), respectively, we get

$$
r_{\mathrm{B} \leftrightarrow \mathrm{X}}(\chi)=r_{\mathrm{V}}+(1-\chi) u_{1}
$$

and

$$
r_{\mathrm{A} \leftrightarrow \mathrm{X}}\left(\chi^{\prime}\right)=r_{\mathrm{V}}+\left(1-\chi^{\prime}\right) u_{2}=r_{\mathrm{V}}+\chi u_{2},
$$

where $r_{\mathrm{V}}$, the nn separation according to the Vegard law, is given by

$$
r_{\mathrm{V}}=(1-\chi) r_{1}+\chi r_{2} .
$$

Substituting (13) and (14) in Eq. (2) we obtain the present expression for $r(\chi)$ as

$$
r(\chi)=r_{\mathrm{V}}+\chi(1-\chi)\left(u_{1}+u_{2}\right)
$$

We have estimated $u_{1}$ and $u_{2}$ from a static Green function model [8] and obtained individual relaxations at different lattice sites over entire composition range from Eqs. (9) and (10) (EXAFS observations) while Eq. (16) gives us the virtual lattice parameter as expected in the X-ray diffraction measurements. The physical content of Eq. (16) may be expressed in the following way: the $\chi(1-\chi)$ dependence enters by the present construction, while the factor $u_{1}+u_{2}$ introduces the relaxation phenomenon into the description.

\subsection{Estimation of relaxation $u$ by a static Green function technique}

In Ref. [8] we developed a static Green function technique to estimate the lattice relaxation. If $u\left(\begin{array}{l}l \\ k\end{array}\right)$ be the relaxation of the $l k$-th ion due to substitution of a defect ion $\mathrm{B}$ in place of an isovalent $\mathrm{A}$ ion in the $\mathrm{AX}$ crystal, and $f\left(\begin{array}{l}l \\ k\end{array}\right)$ be the additional force experienced by the $l k$-th ion due to this replacement, then the new equilibrium demands

$$
f\left[\begin{array}{l}
l \\
k \\
\alpha
\end{array}\right]=\sum_{l^{\prime}, k^{\prime}, \beta} \Phi\left[\begin{array}{ll}
l & l^{\prime} \\
k & k^{\prime} \\
\alpha & \beta
\end{array}\right] u\left[\begin{array}{l}
l^{\prime} \\
k^{\prime} \\
\beta
\end{array}\right]
$$


where $\Phi\left[\begin{array}{cc}l & l^{\prime} \\ k & k^{\prime}\end{array}\right]$ are the force constants of the perfect AX crystal. For a diatomic crystal with $N$ cells, Eq. (17) may be written as

$$
\left(u\left[\begin{array}{l}
l \\
k \\
\alpha
\end{array}\right] \sqrt{m_{k}}\right)=\sum_{l^{\prime}, k^{\prime}, \beta} H^{-1}\left[\begin{array}{cc}
l & l^{\prime} \\
k & k^{\prime} \\
\alpha & \beta
\end{array}\right]\left(f\left[\begin{array}{l}
l \\
k \\
\beta
\end{array}\right] \frac{1}{\sqrt{m_{k^{\prime}}}}\right),
$$

where $m_{k}$ and $m_{k^{\prime}}$ are the masses of the ions of type $k$ and $k^{\prime}$, respectively, and the static Green function $H^{-1}$ is given by

$$
\begin{aligned}
H^{-1} & {\left[\begin{array}{cc}
l & l^{\prime} \\
k & k^{\prime} \\
\alpha & \beta
\end{array}\right]=\sqrt{m_{k} m_{k^{\prime}}} \Phi^{-1}\left[\begin{array}{cc}
l & l^{\prime} \\
k & k^{\prime} \\
\alpha & \beta
\end{array}\right] } \\
& =(1 / N) \sum_{q, j}\left[b(q, k, \alpha, j) b^{*}\left(q, k^{\prime}, \beta, j\right)\right] \frac{\exp \left[\mathrm{i} q\left(r_{k}-r_{k^{\prime}}\right)\right]}{\omega^{2}(q, j)} .
\end{aligned}
$$

In the present paper, we have replaced $\omega^{2}(q, j)$ by an average Einstein frequency defined by

$$
\left\langle\omega^{2}\right\rangle=(1 / 6 N) \sum_{q, j} \omega^{2}(q, j) .
$$

For the $\mathrm{NaCl}$ structure, using a two-body central potential with repulsive interaction $\phi$ up to the nearest neighbour only, we can write the relation between $\left\langle\omega^{2}\right\rangle$ and $G$, the bulk modulus of the host crystal as

$$
\left\langle\omega^{2}\right\rangle=\sum_{k} \frac{1}{m_{k}}\left[\phi^{\prime \prime}(r)+\frac{2 \phi^{\prime}(r)}{r}\right]=\sum_{k} \frac{1}{m_{k}}(3 r G),
$$

where $\phi^{\prime}(t)=\mathrm{d} \phi / \mathrm{d} t$, etc. and $r$ is the nn separation of the host crystal. Finally, applying orthogonality condition for eigenvectors and using Eq. (21) we get the relaxation of the $\mathrm{X}$-ion at the $\mathrm{nn}$ site (100) of the substituted $\mathrm{B}$ ion at (000)

$$
u\left[\begin{array}{c}
100 \\
\alpha
\end{array}\right]=f\left[\begin{array}{c}
k=100 \\
\alpha
\end{array}\right] \frac{1}{m_{100}}\left[\left(\frac{1}{m_{k_{1}}}+\frac{1}{m_{k_{2}}}\right) 3 r G\right]^{-1},
$$

where $k=100$ designates the particular nn ion under consideration and $m_{k_{1}}, m_{k_{2}}$ are the masses of the two ions of the host crystal and $r$ and $G$ are, respectively, the nn separation and bulk modulus of the host crystal.

\section{Results and discussions}

In the previous section we have developed a model which offers a simultaneous explanation to both EXAFS measurements and X-ray diffraction intensity measurements. In Ref. [8] we have found that this model is very successful in explaining EXAFS measurements [2] of nn separations $r_{\mathrm{A} \leftrightarrow \mathrm{X}}$ and $r_{\mathrm{B} \leftrightarrow \mathrm{X}}$ over entire composition range, both for positive ion and negative ion substitutions in case of alkali halide solid solutions. 


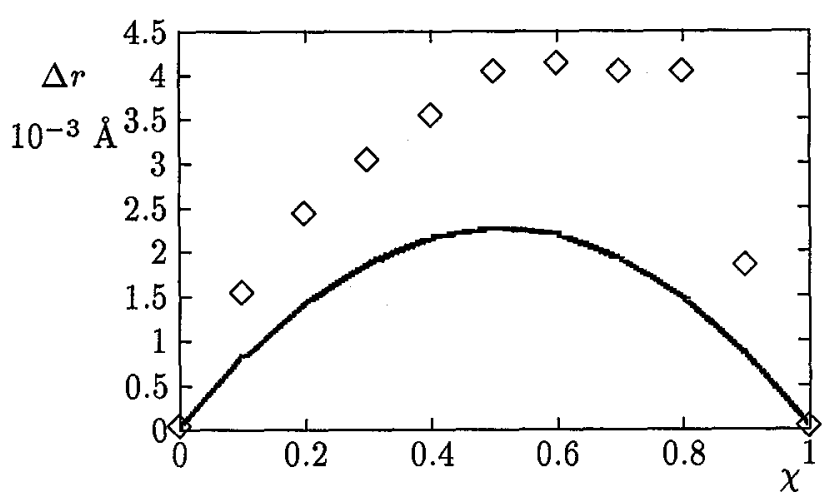

Fig. 1. Comparison of $\Delta r=r(\chi)-r \mathrm{v}$, the deviation of the theoretically calculated values of nn separation $r(\chi)$, from the value suggested by Vegard's law, over the entire composition range to the experimentally observed values for $\mathrm{NaCl}_{1-\chi} \mathrm{Br}_{\chi}$ alloy. Continuous curves are due to the present calculation. Experimental values, shown by squares, are due to Ahtee [12].

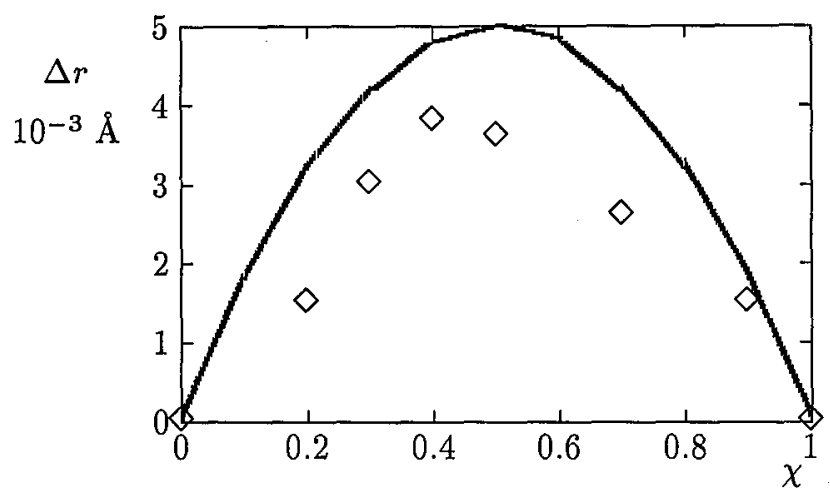

Fig. 2. Comparison of $\Delta r=r(\chi)-r \mathrm{v}$, the deviation of the theoretically calculated values of nn separation $r(\chi)$, from the value suggested by Vegard's law, over the entire composition range to the experimentally observed values for $\mathrm{RbBr}_{1-\chi} \mathrm{I}_{\chi}$ alloy. Continuous curves are due to the present calculation. Experimental values, shown by squares, are due to Ahtee [12].

In the present work, we have calculated $r(\chi)$, the virtual crystal nn separation from Eq. (16) utilising $r_{\mathrm{A} \leftrightarrow \mathrm{X}}$ and $r_{\mathrm{B} \leftrightarrow \mathrm{X}}$ values obtained from Eqs. (13) and (14), respectively. We have presented plots of $r(\chi)-r_{\mathrm{V}}$, the deviation from the Vegard law, over entire composition range for four alkali halide solid solution systems, e.g., $\mathrm{NaCl}_{1-x} \mathrm{Br}_{x}, \mathrm{RbBr}_{1-x} \mathrm{I}_{x}, \mathrm{~K}_{1-x} \mathrm{Rb}_{x} \mathrm{Cl}$ and $\mathrm{K}_{1-x} \mathrm{Rb}_{x}$ Br shown in Figs. 1 to 4 and compared with experimental values obtained from $\mathrm{X}$-ray diffraction measurements. Considering the uncertainties in measurements and also in input data, the agreement seems to be quite satisfactory. It is significant to note that all available measurements on alkali halide solid solutions show that the dependence of $r(\chi)$ on $\chi$ is not linear in nature as suggested by Vegard's law. The present model correctly obtains the variation of $r(\chi)$ with $\chi$ to be of quadratic nature. This model 


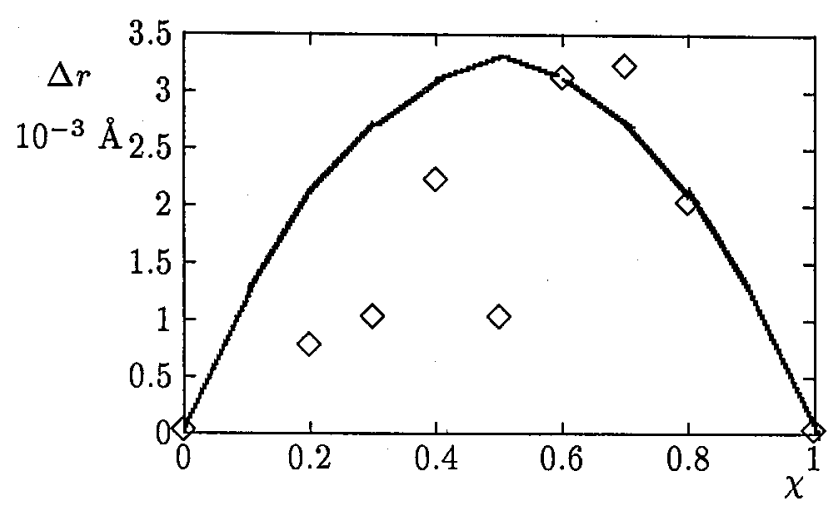

Fig. 3. Comparison of $\Delta r=r(\chi)-r_{\mathrm{V}}$, the deviation of the theoretically calculated values of $n n$ separation $r(\chi)$, from the value suggested by Vegard's law, over the entire composition range to the experimentally observed values for $\mathrm{K}_{1-\chi} \mathrm{Rb}_{\chi} \mathrm{Cl}$ alloy. Continuous curves are due to the present calculation. Experimental values, shown by squares, are due to Ahtee [12].

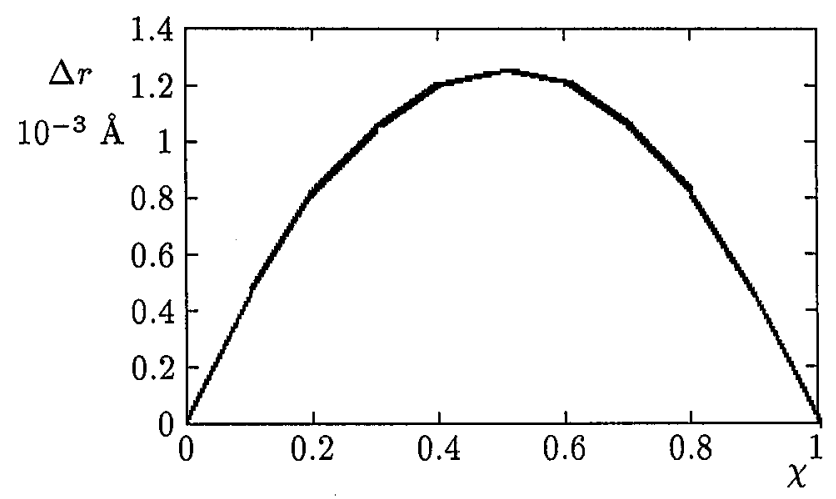

Fig. 4. Comparison of $\Delta r=r(\chi)-r_{\mathrm{V}}$, the deviation of the theoretically calculated values of $n$ n separation $r(\chi)$, from the value suggested by Vegard's law, over the entire composition range to the experimentally observed values for $K_{1-\chi} R b_{\chi} \mathrm{Br}$ alloy. Continuous curves are due to the present calculation. Experimental values are not a vailable.

also predicts the sign of the deviation $r(\chi)-r_{\mathrm{V}}$ correctly. In case of alkali halides, all available measurements show $r(\chi)-r_{\mathrm{V}}$ to be positive as obtained in the present derivation. But this test is very crucial for metallic solid solutions where in some cases (e.g. Au-Ag solid solution [11]) this is found to be negative experimentally. It is significant that our present model also predicts that the sign of $r(\chi)-r_{\mathrm{V}}$ is negative for this case.

As can be seen from Eq. (16), the model is very sensitive to the dilute relaxation parameters $u_{1}$ and $u_{2}$, obtained for a $\mathrm{B}$ ion substitution in AX host crystal and an A ion substitution in BX host crystal, respectively. The static Green function technique developed is found to be a quite dependable one for alkali halide solid solutions [8] and metallic solid solutions [9]. We present a list of $u_{1}$ and $u_{2}$, 
for the four cases studied, in Table. We do not, however, present $r_{\mathrm{A} \leftrightarrow \mathrm{X}}(\chi)$ and $r_{\mathrm{B} \leftrightarrow \mathrm{X}}(\chi)$ since this has already been presented in [8]. Of the two possible values of $r_{\mathrm{B} \leftrightarrow \mathrm{X}}$, one with $r_{\mathrm{A} \leftrightarrow \mathrm{X}}$ as host crystal and the other with $r_{\mathrm{B} \leftrightarrow \mathrm{X}}$ as the host crystal in an $\mathrm{A}_{1-\chi} \mathrm{B}_{\chi} \mathrm{X}$ solid solution, we preferred taking the first choice, i.e. $r_{\mathrm{B} \leftrightarrow \mathrm{X}}$ from $\mathrm{AX}$ host crystal and $r_{\mathrm{A} \leftrightarrow \mathrm{X}}$ from $\mathrm{BX}$ host crystal, respectively. The values from the alternative choices are very close, but for $r(\chi)-r v$ sensitivity requirement being very stringent, we choose the first alternative. This is to avoid any arbitrariness involved in introducing Huang's idea of uniform elastic dilation at high concentration of defect. An appropriate choice of defect strength constant $K$, present in the Huang expression can only remove this arbitrariness. We refer to our detailed discussion [7] on the defect strength constant $K$ and write

$$
K=k r_{0}^{2} u,
$$

where $k$ is determined from the smooth merging of the microscopic relaxation $(u)$ experienced by the nearest neighbour to the defect, to the macroscopic strain developed in the rest of the crystal. For an AX host crystal, we have determined $k_{1}$ from the condition of smooth merging of $r_{\mathrm{B} \leftrightarrow \mathrm{X}}$ to $r_{\mathrm{BX}}$ at $\chi=1$ (see Eq. (11)). Similarly, for BX host crystal smooth merging of $r_{A \leftrightarrow X}$ to $r_{\mathrm{AX}}$ at $\chi^{\prime}=1$ (i.e. at $\chi=0)$.

\section{TABLE}

Calculated values of relaxations of ions at nearest neighbour sites of the defect ion for dilute defect concentration. The relaxation is given in units of $10^{-8} \mathrm{~cm}$.

\begin{tabular}{c|c|c}
\hline System & $\begin{array}{c}\text { Relaxation } \\
\text { (theoretical) } \\
{\left[10^{-8} \mathrm{~cm}\right]}\end{array}$ & $\begin{array}{c}\text { Experiment } \\
\text { (EXAFS) } \\
{[2]}\end{array}$ \\
\hline $\mathrm{Br}^{-}$in $\mathrm{NaCl}$ & 0.123 & - \\
$\mathrm{Cl}^{-}$in $\mathrm{NaBr}$ & -0.114 & - \\
$\mathrm{I}^{-}$in $\mathrm{RbBr}$ & 0.160 & $0.16 \pm 0.02$ \\
$\mathrm{Br}^{-}$in $\mathrm{RbI}$ & -0.140 & $-0.15 \pm 0.02$ \\
$\mathrm{Rb}^{+}$in $\mathrm{KBr}$ & 0.070 & $0.07 \pm 0.005$ \\
$\mathrm{~K}^{+}$in $\mathrm{RbBr}$ & -0.065 & $-0.065 \pm 0.005$ \\
$\mathrm{Rb}^{+}$in $\mathrm{KCl}$ & 0.099 & - \\
$\mathrm{K}^{+}$in $\mathrm{RbCl}$ & -0.086 & -
\end{tabular}

We had to choose solid solutions depending on the availability of X-ray diffraction measurement. Isovalent positive ion substitution is examined in case of $\mathrm{KCl}-\mathrm{RbCl}$ and $\mathrm{KBr}-\mathrm{RbBr}$ solid solutions and isovalent negative ion substitution is examined of $\mathrm{NaCl}-\mathrm{NaBr}$ and $\mathrm{RbBr}-\mathrm{RbI}$ solid solutions. Of these EXAFS measurements are available only for two cases, $\mathrm{KBr}-\mathrm{RbBr}$ and $\mathrm{RbBr}-\mathrm{RbI}$. 
It is interesting to note that in obtaining $r(\chi), r_{\mathrm{A} \leftrightarrow \mathrm{X}}, r_{\mathrm{B} \leftrightarrow \mathrm{X}}$ over the entire composition range no particular behaviour of the solid solution is used as input.

The model is quite simple but seems to be fairly useful, particularly, its possible utility in case of two-phase solid solution with heterostructure solutes, approximated as isotropic solid, keeps it worth pursuing.

\section{Acknowledgment}

One of us (S.K.D.) is grateful to Prof. (Dr.) Abhijeet Mookerjee, S.N. Bose National Centre for Basic Sciences, JD Block, Sector 3, Salt Lake, Calcutta 700091, for fruitful discussion.

\section{References}

[1] L. Vegard, Z. Phys. 5, 17 (1921).

[2] J.B. Boyce, J.C. Mikkelson Jr., Phys. Rev. B 31, 6903 (1985).

[3] G.S. Durham, J.A. Hawkins, J. Chem. Phys. 19, 149 (1951).

[4] J.R. Hardy, J. Phys. Chem. Solids 15, 39 (1960); J. Phys. Chem. Solids 29, 2009 (1968).

[5] Y. Fukai, J. Phys. Soc. Jpn. 18, 1413 (1963).

[6] D.L. Fancher, G.R. Barsch, J. Phys. Chem. Solids 30, 2503 (1969).

[7] S. Paul, S. Sengupta, Phys. Status Solidi B 68, 703 (1975); 83, 645 (1977).

[8] S. Datta Roy (Paul), S. Sengupta, Phys. Status Solidi B 162, 89 (1990).

[9] S.K. Das, S. Datta Roy (Paul), Acta Phys. Pol. A 95, 359 (1999).

[10] K. Huang, Proc. R. Soc. Lond. A 190, 102 (1947).

[11] C.S. Barrett, Structure of Metals, 2nd ed., McGraw-Hill, New York 1952, p. 222.

[12] M. Ahtee, Ann. Acad. Sci. Fennica A 6, 1 (1969). 